\title{
Responsiveness and Construct Validity of a Symptom Scale for Acute Otitis Media
}

\author{
Nader Shaikh, MD, MPH, * Alejandro Hoberman, MD, * Jack L. Paradise, MD, * Howard E. Rockette, PhD, $\dagger$ \\ Marcia Kurs-Lasky, MS, $†$ D. Kathleen Colborn, BS,* Diana H. Kearney, RN, CCRC,* and \\ Lisa M. Zoffel, CRNP*
}

\begin{abstract}
Background: Because resolution of symptoms is a primary goal of antimicrobial therapy in children with acute otitis media (AOM), measurement of symptoms in studies of antimicrobial effectiveness in such children is important. We have developed a scale for measuring symptoms of AOM in young children (AOM-SOS), and we present data on its construct validity and responsiveness.

Methods: We followed children 3 months to 3 years of age with AOM, who were receiving antimicrobial treatment, using the AOM-SOS scale. The scale was administered at the enrollment visit, as a twice-a-day diary measure, and at the follow-up visit (days 5-7). To evaluate construct validity, we examined the correlation, at entry, between AOM-SOS scores and scores on other measures of pain and functional status. To evaluate the scale's responsiveness, we examined the change in scale scores from entry to follow-up. We also examined the levels of agreement between the scale scores and overall assessments of the children by parents.

Results: We enrolled 70 children (mean age 12.5 months) of whom 57 returned for follow-up. The magnitude of the correlations between the AOM-SOS scale scores and other measures of pain and functional status ranged from 0.56 to 0.84 . The responsiveness of the AOM-SOS, as measured by the standardized response mean was 1.20 .

Conclusions: These data support the validity and responsiveness of the AOM-SOS; the scale seems to measure effectively both pain and overall functional status in young children with AOM. Changes in score over the first few days of illness were substantial and generally matched the assessments both of parents and of clinicians. The AOM-SOS promises to be useful as an outcome measure in clinical studies of AOM.
\end{abstract}

Key Words: acute otitis media, validation study, patient-reported outcome measure, pediatrics

(Pediatr Infect Dis J 2009;28: 9-12)

A growing body of evidence suggests that using surrogate outcomes as sole measures of efficacy in clinical trials is not sufficient. ${ }^{1}$ Surrogate outcomes refer to test results that are used in place of clinical outcomes in trials of efficacy. Although often easy to measure, surrogate outcomes frequently lack direct clinical relevance. $^{2}$

In clinical trials of antimicrobial efficacy in children with acute otitis media (AOM), eradication of bacteria from middle ear

Accepted for publication July 3, 2008.

From the *Division of General Academic Pediatrics, Children's Hospital of Pittsburgh, University of Pittsburgh School of Medicine, Pittsburgh, PA; and $\dagger$ Department of Biostatistics, University of Pittsburgh Graduate School of Public Health, Pittsburgh, PA

Supported by funds from Department of Pediatrics, University of Pittsburgh.

Address for correspondence and for research use of the AOM-SOS: Nader Shaikh,

MD, MPH, Children's Hospital of Pittsburgh, General Academic Pediatrics,

3705 Fifth Ave, Pittsburgh, PA 15213-2583. E-mail: nader.shaikh@chp.edu.

Copyright (C) 2008 by Lippincott Williams \& Wilkins

ISSN: 0891-3668/09/2801-0009

DOI: $10.1097 /$ INF.0b013e318185a3a0 fluid has frequently served as the sole outcome measure. ${ }^{3}$ However, because resolution of symptoms is a primary goal of antimicrobial therapy, and because bacteriologic outcomes do not accurately predict symptomatic outcomes, $2,4,5$ measurement of symptoms should logically be part of all efficacy trials. That the measurement of symptoms has often not been undertaken has been attributable in part to the lack of a properly validated outcome measure.

We have developed an outcome instrument that measures symptoms of AOM in young children: the acute otitis media severity of symptom scale (AOM-SOS). In developing the instrument we used parent questionnaire, review of the literature, and consultation with experts. In a preliminary study, we showed that AOM-SOS scores correlated well with otoscopic diagnosis (ie, either AOM, otitis media with effusion, or normal middle ear status), and that scores changed as expected with changes in clinical status. ${ }^{6}$

In the present study, we used a revised version of the AOM-SOS, which differed from the version used in our preliminary study in that we separated the single question concerning irritability (ie, fussiness or increased crying) into separate components, namely, irritability, and increased crying. In addition, instead of using the recorded temperature, we asked parents to estimate the degree of fever (none, a little, or a lot). We evaluated: (1) whether the AOM-SOS as revised was adequately responsive for use as a clinical end point in AOM trials; (2) whether AOM-SOS scores correlated with other measures of pain and quality of life; (3) whether changes in AOM-SOS scores correlated with assessments by parents; and (4) whether parents had difficulty in interpreting either the instructions for using the instrument or the instrument's contents.

\section{METHODS}

We enrolled consecutive children aged 3 months to 3 years presenting with AOM to a general ambulatory pediatric clinic at Children's Hospital of Pittsburgh between November 2005 and May 2006. Our criteria for diagnosing AOM consisted of (1) the presence of bulging or distinct erythema of the tympanic membrane (TM); and (2) middle ear effusion, as evidenced either by the presence of at least 2 of 3 TM findings, namely, opacity, yellow or white discoloration, or decreased mobility, or by the presence of air-fluid interfaces. We excluded children who were asymptomatic or who had tympanostomy tubes in place, otorrhea, or a TM perforation, as well as children about to undergo diagnostic tympanocentesis. All children were treated with antimicrobials chosen by their respective primary-care providers. The study-team otoscopists (A.H., N.S., L.Z.) were not blinded to children's symptoms. The investigators had extensive experience in conducting AOM-related clinical research. There was good interobserver agreement between the 3 otoscopists; $\kappa$ values derived from diagnoses registered in a 50 -ear video-otoscopic test ranged from 0.78 to 0.81 .

From the date of enrollment (day 1) we followed children with AOM for 5-7 days. At both the enrollment visit and the follow-up visit (days 5-7), we interviewed parents regarding their child's symptoms by reading verbatim the questions on the AOM- 
SOS. We then performed otoscopic examination and asked parents to complete 3 reference measures:

- A 10-point pain scale in which parents rated the severity of their child's pain from 0 ("no pain") to 10 ("worst possible pain");

- The Pediatric Postoperative Pain Measure for Parents, in which parents assessed the presence or absence of 15 nonverbal painrelated behaviors. Higher scores indicate greater degrees of pain. This scale was developed for use in children 1-15 years of age, ${ }^{7}$ and seemed appropriate for use in this study; and

- The Functional Status Questionnaire, ${ }^{8}$ a 14-item scale that measures overall health status in children $0-16$ years of age. The questionnaire asks parents about the presence or absence of key behaviors over the preceding 2-week period. Higher scores indicate more favorable status. For the present study, we modified the questionnaire to ask only about the preceding day while leaving unchanged the wording, sequence, and number of questions.

During the interval between the enrollment (day 1) and the follow-up visit, we asked parents to complete the AOM-SOS twice daily, and also to rate whether, on the morning of day 2, compared with the time of enrollment, their child was "a lot better," "a bit better," "the same," "a bit worse," or "a lot worse."

To evaluate how parents interpreted questions on the AOMSOS, we conducted face-to-face semi-structured interviews with 10 parents at the follow-up visit. In these interviews, we questioned parents regarding any difficulties in understanding the instructions, in understanding the qualifier "more than usual," in understanding the meaning of each symptom, or in completing diaries. We asked the parents to elaborate on their answers and we recorded their responses verbatim.

To estimate cross-sectional construct validity, we examined the correlation between AOM-SOS scores and scores on reference measures at the enrollment visit. To determine longitudinal construct validity, we compared changes in AOM-SOS scores (from day 1 to days 5-7) with changes in reference measure scores. We had hypothesized, a priori, a correlation of 0.5 between changes in AOM-SOS scores and changes in scores on measures of pain and a correlation of -0.6 between changes in AOM-SOS scores and changes in scores on the functional status measure.

To evaluate responsiveness-the ability of the instrument's scores to change in conjunction with changes in clinical status-we examined the change in AOM-SOS scores from enrollment to the follow-up visit. Generally, an instrument is considered responsive when the mean change in scores is large relative to the scores' variability. We calculated the standardized response mean (SRM) by dividing the magnitude of the mean change in score by the standard deviation of the change. An SRM $>0.5$ usually indicates good responsiveness. $^{9-11}$

All questions on the AOM-SOS, with the exception of the question about ear pain, ask parents about directly observable behaviors. The question about ear pain, in contrast, asks parents to interpret and synthesize information from a variety of sources, and may accordingly be more prone to variability. We therefore explored the psychometric ramifications of removing this question from the instrument, and found no appreciable difference in results whether the ear pain question was or was not included (details are available from the authors on request). The results presented here are those obtained with the question excluded.

All analyses were performed using 2-tailed tests, with statistical significance set at $P<0.05$. We used $\chi^{2}$ tests to evaluate differences in proportions, the $t$ test to test for differences between mean results, and present Pearson correlation coefficients.

\section{RESULTS}

We enrolled 70 children with AOM (mean age, 12.5 months; range, 3-28 months). Selected demographic and clinical characteristics of the children are shown in Table 1. All children were symptomatic (mean AOM-SOS score $=6.39$ ). A total of 57 children returned for their follow-up evaluation; mean time to follow-up was 6.1 days. There were no significant differences in race, gender, or age between children who returned for follow-up and those who did not. Parents had little difficulty completing the AOM-SOS at home; of the 475 questionnaires received, only 8 (1.7\%) had any missing data.

\section{Construct Validity}

Table 2 shows the correlations between AOM-SOS scores and scores on reference measures at the time of enrollment, and the correlations between changes in AOM-SOS scores from day 1 to days 5-7 and changes in scores on those reference measures in that interval. The magnitude of the correlations ranged in the expected directions from 0.56 to 0.84 and were similar to the values we had predicted a priori.

\section{Responsiveness}

Mean AOM-SOS scores at the baseline and at follow-up visits (days 5-7) were 6.39 and 1.34, respectively. Day-to-day changes in AOM-SOS scores are shown in Figure 1. The SRM was 1.20. Seven children had an AOM-SOS score that was unchanged or higher at the follow-up compared with the baseline visit. Of these, 2 had developed an intercurrent illness ( 1 with exudative pharyngitis and 1 with viral upper respiratory tract infection) and 2 had persistently bulging tympanic membranes.

\section{Parental Assessment}

As shown in Table 3, we noted good overall agreement between change in AOM-SOS scores and parents' assessment of change from day 1 to day 2 . Among the 19 children who were

TABLE 1. Distribution of Children According to Selected Demographic and Clinical Characteristics

\begin{tabular}{|c|c|}
\hline Characteristic & $\begin{array}{l}\text { No. }(\%) \text { Children } \\
\quad(\mathrm{n}=70)\end{array}$ \\
\hline \multicolumn{2}{|l|}{ Age at entry, months } \\
\hline $3-6$ & $12(17)$ \\
\hline $7-12$ & $29(41)$ \\
\hline $13-18$ & $19(27)$ \\
\hline $19-24$ & $7(10)$ \\
\hline $25-30$ & $3(4)$ \\
\hline \multicolumn{2}{|l|}{ Gender } \\
\hline Male & $39(56)$ \\
\hline Female & $31(44)$ \\
\hline \multicolumn{2}{|l|}{ Race } \\
\hline White & $22(31)$ \\
\hline African American & $44(63)$ \\
\hline Other & $4(6)$ \\
\hline \multicolumn{2}{|l|}{ Maternal education } \\
\hline Less than high school & $7(10)$ \\
\hline High school graduate/GED & $48(69)$ \\
\hline College graduate & $15(21)$ \\
\hline \multicolumn{2}{|l|}{ Health insurance } \\
\hline Private & $13(19)$ \\
\hline Public & $56(80)$ \\
\hline None & $1(1)$ \\
\hline \multicolumn{2}{|l|}{ Day care ( $>4 \mathrm{~h}$ a week) } \\
\hline Yes & $27(39)$ \\
\hline No & $43(61)$ \\
\hline \multicolumn{2}{|c|}{ Current upper respiratory infection } \\
\hline Yes & $66(94)$ \\
\hline No & $4(6)$ \\
\hline
\end{tabular}


TABLE 2. Correlations Between AOM-SOS Scores and Scores on Reference Measures at Enrollment (Day 1), and Correlations Between Changes (From Enrollment to Follow-Up on Day 5-7) in AOM-SOS Scores and Changes in Reference Measure Scores

\begin{tabular}{ccc}
\hline \hline & \multicolumn{2}{c}{ Correlations } \\
\cline { 2 - 3 } $\begin{array}{c}\text { Reference } \\
\text { Measure }\end{array}$ & $\begin{array}{c}\text { AOM-SOS Scores } \\
\text { and Reference } \\
\text { Measure Scores } \\
\text { (Day 1) }\end{array}$ & $\begin{array}{c}\text { Changes in AOM-SOS Scores } \\
\text { and Changes in Reference } \\
\text { Measure Scores } \\
\text { (Day 1 to Day 5-7) }\end{array}$ \\
\hline $\begin{array}{c}\text { Pain rating scale } \\
\begin{array}{c}\text { Postoperative } \\
\text { pain scale }\end{array}\end{array}$ & 0.56 & 0.60 \\
Functional & 0.82 & 0.85 \\
status scale & -0.83 & -0.84 \\
\hline
\end{tabular}

considered "a bit better" by their parents, AOM-SOS scores decreased by a mean of 4.2 points. This can be used as a rough estimate for the minimal important difference. We are currently conducting a large study using the AOM-SOS, which will allow us to more precisely estimate the minimal important difference.

\section{Parents' Understanding and Use of the AOM-SOS (Cognitive Debriefing)}

None of the 10 parents interviewed reported any difficulties in understanding either the instructions or the meaning of the word "usual." Except for the question on ear pain, all of the items on the instrument were regarded as "very easy" or "easy" to understand by
TABLE 3. Change in AOM-SOS Score from Day 1 to Day 2 in Relation to Parental Assessment of Change in Overall Status from Day 1 to Day 2

\begin{tabular}{|c|c|c|c|c|c|}
\hline \multirow{2}{*}{$\begin{array}{l}\text { Change in } \\
\text { AOM-SOS Score }\end{array}$} & \multicolumn{5}{|c|}{ Parental Assessment of Status on Day $2^{*}$} \\
\hline & $\begin{array}{l}\text { A Lot } \\
\text { Worse }\end{array}$ & $\begin{array}{l}\text { A Bit } \\
\text { Worse }\end{array}$ & $\begin{array}{c}\text { The } \\
\text { Same }\end{array}$ & $\begin{array}{l}\text { A Bit } \\
\text { Better }\end{array}$ & $\begin{array}{l}\text { A Lot } \\
\text { Better }\end{array}$ \\
\hline-9 & & & & & \\
\hline-8 & & 1 & & & \\
\hline-6 & 1 & & & & \\
\hline-2 & & 3 & & & \\
\hline-1 & & & 2 & & \\
\hline 0 & & 1 & 2 & 1 & \\
\hline 1 & & & 4 & 3 & \\
\hline 2 & & & 3 & & 2 \\
\hline 3 & & & 2 & 2 & 1 \\
\hline 4 & & & & 5 & \\
\hline 5 & & & 1 & 5 & \\
\hline 6 & & & & & 2 \\
\hline 7 & & & & 1 & 1 \\
\hline 8 & & & & 1 & \\
\hline 10 & & & & 1 & \\
\hline
\end{tabular}

9 of the 10 parents; however, 5 of the 10 parents had difficulty with the ear pain item. In most of these instances, parents reported that they were unable to determine with certainty whether their child was experiencing ear pain. Tugging, rubbing, holding, or pulling at the ear, however, seemed to be well understood by most parents; when asked to explain the meaning of such behavior, 8 of the 10 parents associated it with ear pain.

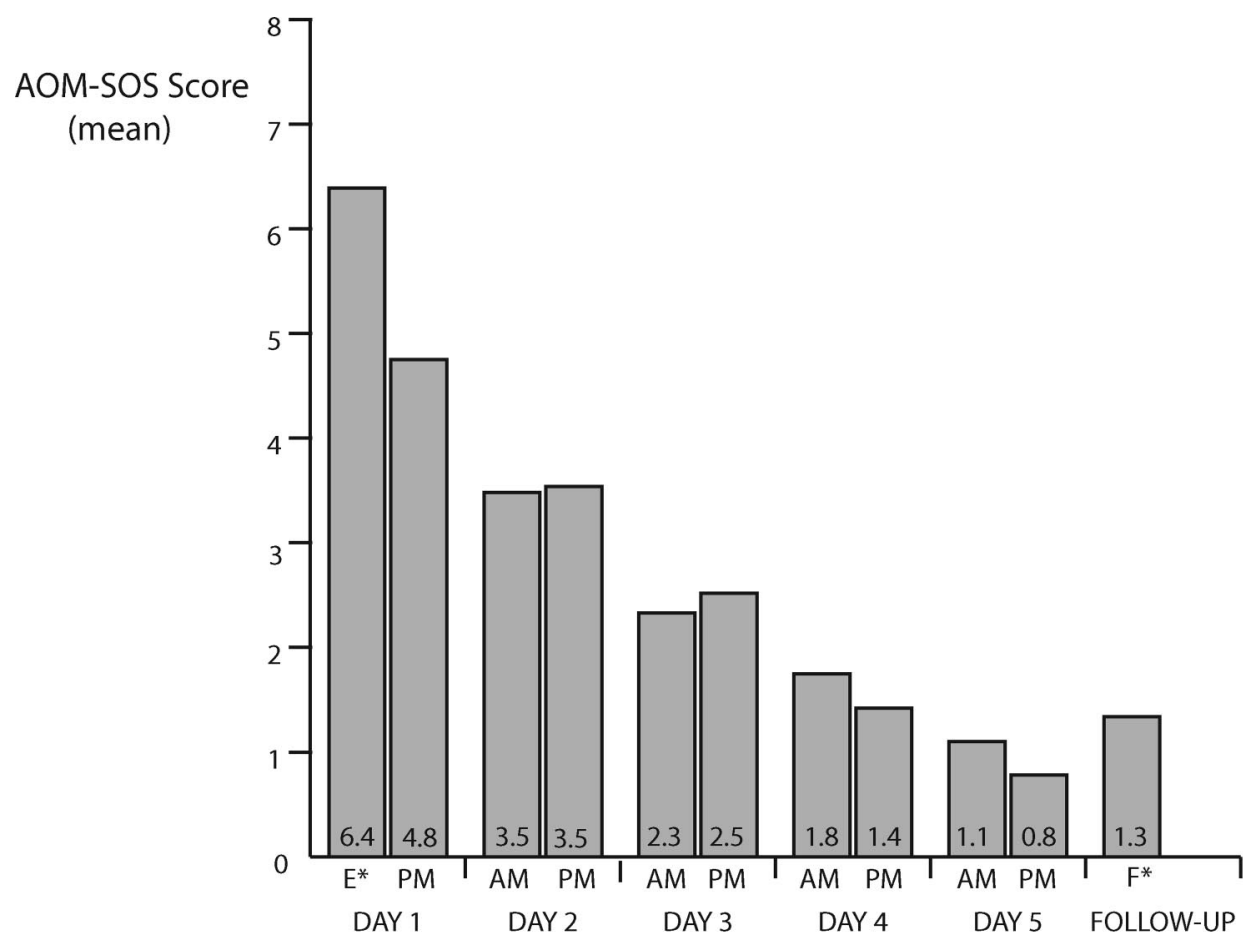

${ }^{*}$ Assessments at the enrollment $(E)$ and follow-up visits $(F)$ were performed by interview; all other assessments were performed by parents and recorded in diaries.

FIGURE 1. Day-to-day change in mean AOM-SOS scores in 70 children with AOM receiving antimicrobial therapy. 
TABLE 4. AOM-SOS (Version 3.0)*

We are interested in finding out how your child has been doing. For each question, please place a check mark in the box corresponding to your child's symptoms. Please answer all questions.

Over the past $12 \mathrm{~h}$, has your child been tugging, rubbing, or holding the ear(s) more than usual?

Over the past $12 \mathrm{~h}$, has your child been crying more than usual?

Over the past $12 \mathrm{~h}$, has your child been more irritable or fussy than usual?

Over the past $12 \mathrm{~h}$, has your child been having more difficulty sleeping than usual?

Over the past $12 \mathrm{~h}$, has your child been less playful or active than usual?

Over the past $12 \mathrm{~h}$, has your child been eating less than usual?

Over the past $12 \mathrm{~h}$, has your child been having fever or feeling warm to touch?

*Reprinted with permission. (C) 2008, University of Pittsburgh. All Rights Reserved.

\section{DISCUSSION}

In a previous study, we described the development and preliminary evaluation of a parent-reported symptom instrument for children with $\mathrm{AOM}^{6}{ }^{6}$ In the present report, we present additional data on the responsiveness and validity of the AOM-SOS. We have shown that the day-to-day responsiveness of the AOM-SOS suggests it as a potentially useful tool in following the symptom burden of AOM in clinical trials. Further, we have shown that the instrument correlates well with results of other measures of pain and functional status. Accordingly, mean change in AOM-SOS scores from baseline to follow-up could be used as one measure of improvement or deterioration. Although variability exists in how individual children respond to $\mathrm{AOM}$ and in how parents perceive and report their child's AOM symptoms, measurement error would be lessened because each child acts as his or her own control.

Importantly, our results do not suggest using the AOM-SOS score alone at any stage of AOM for determining the severity of the illness or for managing individual cases. Rather, we propose 2 ways to use AOM-SOS scores. In the first approach, time to achievement of a specified score would be compared between treatment groups. In the second approach, mean change in score would be compared between treatment groups at one or more designated time points. Here, the minimally important difference - the change in score that parents endorse as important-could be used to interpret results. ${ }^{12-14}$

We have slightly modified the AOM-SOS based on findings of this study. First, we removed the question about ear pain from the instrument in response to some parents' difficulty in interpreting this item and because removal of this item did not seem to affect the overall performance of the instrument. Second, in an effort to reduce variability in responses regarding time frame being assessed, we have decided to incorporate the phrase "over the past 12 hours" in each question (version 3.0, Table 4).

A limitation of the present study is that the AOM-SOS was administered in 2 ways: by direct questioning by investigators at enrollment and follow-up visits and by parents recording responses in a written diary at all intermediate measurement points. Some variability may have thereby been introduced; direct questioning seemed to have resulted in slightly higher symptom scores. Using a single method of administration, either interview or self-report, might therefore be preferable in future studies.

In summary, this study provides additional data on the validity and responsiveness of the AOM-SOS, and further supports its use as a measure of outcome in clinical studies of AOM in young children.

\section{REFERENCES}

1. Guidance for industry: patient-reported outcome measures: use in medical product development to support labeling claims: draft guidance. Health Qual Life Outcomes. 2006;4:79.

2. Powers JH, Zalkikar J, Johann-Liang R. Correlation between bacteriologic eradication and clinical cure in acute otitis media. Pediatr Infect Dis J. 2004;23:976 [author reply 976-978].

3. Dagan R, Leibovitz E, Greenberg D, et al. Early eradication of pathogens from middle ear fluid during antibiotic treatment of acute otitis media is associated with improved clinical outcome. Pediatr Infect Dis J. 1998;17:776782 .

4. Johann-Liang R, Zalkikar J, Powers JH. Correlation between bacteriologic and clinical endpoints in trials of acute otitis media. Pediatr Infect Dis J. 2003;22:936-937 [author reply 937].

5. Marchant CD, Carlin SA, Johnson CE, et al. Measuring the comparative efficacy of antibacterial agents for acute otitis media: "the Pollyanna phenomenon." J Pediatr. 1992;120:72-77.

6. Shaikh N, Hoberman A, Paradise JL, et al. Development and preliminary evaluation of a parent-reported outcome instrument for clinical trials in acute otitis media. Pediatr Infect Dis J. 2008;28:5-8.

7. Chambers CT, Reid GJ, McGrath PJ, et al. Development and preliminary validation of a postoperative pain measure for parents. Pain. 1996;68:307313 .

8. Stein RE, Jessop DJ. Functional status II(R). A measure of child health status Med Care. 1990;28:1041-1055.

9. Deyo RA, Diehr P, Patrick DL. Reproducibility and responsiveness of health status measures. Statistics and strategies for evaluation. Control Clin Trials. 1991;12(4 Suppl):142S-158S.

10. Guyatt G, Walter S, Norman G. Measuring change over time: assessing the usefulness of evaluative instruments. J Chronic Dis. 1987;40:171178 .

11. Guyatt GH, Bombardier C, Tugwell PX. Measuring disease-specific quality of life in clinical trials. Can Med Assoc J. 1986;134:889895.

12. Juniper EF, Guyatt GH, Willan A, et al. Determining a minimal importan change in a disease-specific Quality of Life Questionnaire. J Clin Epidemiol. 1994;47:81-87.

13. Jaeschke R, Singer J, Guyatt GH. Measurement of health status. Ascertaining the minimal clinically important difference. Control Clin Trials. 1989;10: 407-415.

14. Revicki DA, Cella D, Hays RD, et al. Responsiveness and minimal important differences for patient reported outcomes. Health Qual Life Outcomes. $2006 ; 4: 70$ 